\title{
Landbesitzunsicherheit - Bedeutung für die Bodendegradation in Entwicklungsländern
}

\section{Einleitung}

Dieser Beitrag beschäftigt sich mit dem Problem der Bodendegradation in Entwicklungsländern aus der Perspektive der Eigentumsrechts- und Umweltökonomie. Die Umweltökonomie ist ja für die Geographie, welche sich u. a. auch mit Mensch-Umwelt-Beziehungen auseinandersetzt, von besonderer Bedeutung. Der Eigentumsrechtsansatz in der Umweltökonomie besagt, daß fehlende, abgeschwächte oder nicht durchsetzbare Eigentumsrechte bezüglich Umweltgütern, wie z. B. Boden, den Hauptgrund für Umweltdegradation darstellen (BUHBE 1985). Es wird vorgeschlagen, Eigentumsrechte zu stärken oder - wo sie fehlen - zuzuweisen, und es wird angenommen, daß damit den Besitzern der Umweltgüter Anreize vermittelt werden, zu ihren Umweltgütern Sorge zu tragen. Es ist naheliegend, diesen Ansatz für die Erklärung von Bodendegradation in Entwicklungsländern zu wählen, weil hier die Landrechte häufig - so wird zumindest vermutet - unklar und umstritten sind und weil oft Besitzdokumente (Landtitel) fehlen (JOHNSON 1972, SOUTHGATE 1988, 5-8).

Allerdings sind eigentumsrechtsökonomische Analysen von Bodendegradation in Entwicklungsländern oft deduktiv und abstrakt, und sie widerspiegeln die realen Bedingungen in Entwicklungsländern nur ungenau. Andererseits gibt es viele empirische Studien, die sich mit Grundbesitzstrukturen in verschiedenen Entwicklungsregionen, in verschiedenen Agrarsystemen oder in verschiedenen Agrarstrukturen beschäftigen. Diese empirischen Studien kommen of zu sehr unterschiedlichen Schlußfolgerungen sowohl, was die Analyse der Problematik, als auch, was Politikeingriffe betrifft.

Dieser Beitrag faßt einige zentrale Aspekte einer Studie zusammen, die der Autor im Rahmen eines Forschungsaufenthaltes bei der "Environmental Policy and Research Division» der Weltbank in Washington D. C. und am Land Tenure Center der University of WisconsinMadison erarbeitete und deren Ziel es war, die eigentumsrechtsökonomische Literatur mit den verschiedenen Bereichen empirischer Literatur zu verknüpfen (WACHTER 1992). In der Studie wurde einerseits eine Typologie von Problemsituationen bezüglich Besitzunsicherheit erarbeitet, und andererseits wurde untersucht, ob Landtitulierung zur Erhöhung der Besitzsicherheit und zur Verminderung von Bodendegradationsproblemen beitragen könnte.

\section{Eigentumsrechte und Bodendegradation}

Unter Bodendegradation ist eine Vielzahl von Phänomenen zu subsumieren, die durch Übernutzung, Fehlnutzung und fehlenden Unterhalt entstehen können, wie z. B. Überweidung, Erosion, Bodenversauerung, Versalzung, Überdüngung, Verfall von Bewässerungssystemen, Terrassen usw. Unter Bodendegradation sind alle Prozesse zu verstehen, die die Ertragsfähigkeit des Bodens vermindern, wobei es hier allein um die Degradation von Agrarland geht (CHISHOLM / DUMSDAY 1987).

Das Wesen der Bodenkonservierung kann dagegen wie folgt dargestellt werden. Bodenschutz ist eine Langzeitangelegenheit und hat Investitionscharakter. Man verzichtet heute auf einen maximalen Ertrag und nimmt Aufwendungen auf sich, um in Zukunft über einen höheren Ertrag zu verfügen (Abb. 1).

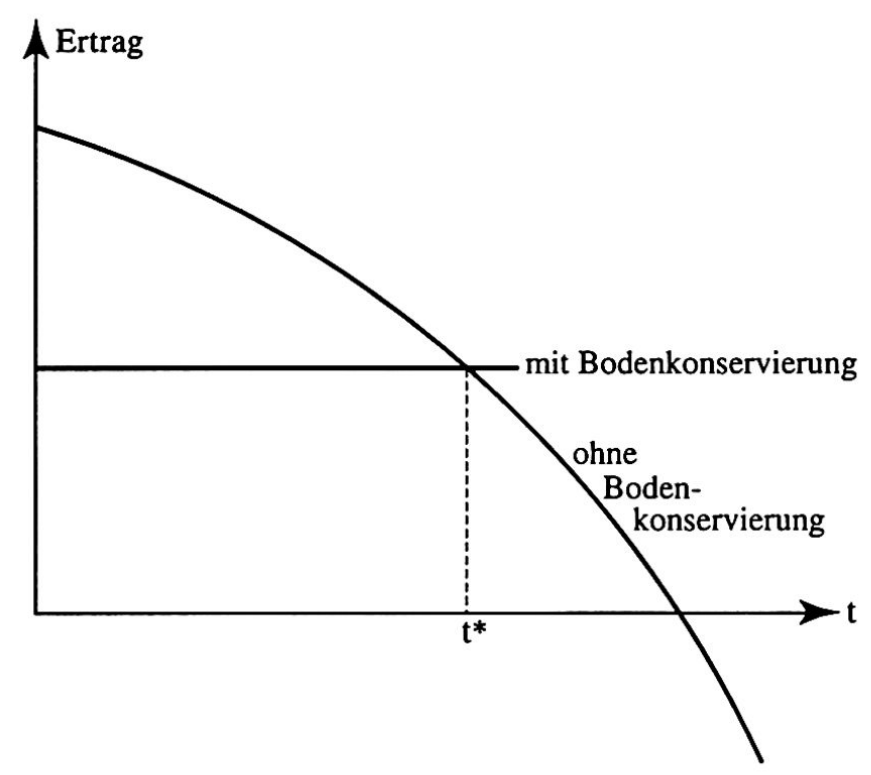

Abb.1 Bodenertrag mit und ohne Bodenkonsenvierung (Quelle: ANDERSON / THAMPAPILLAI [1990, 10]).

Daniel Wachter, PD Dr., Geographisches Institut der Universität Zürich, Winterthurerstraße 190, 8057 Zürich 
Was die Bedeutung von Eigentumsrechten für Investitionen in Bodenschutz und landwirtschaftliche Produktivität betrifft, werden in der Literatur im allgemeinen zwei wichtige Aspekte erwähnt (FEDER / FEENY 1991): Handlungsanreize und Zugang zu Kredit. Bezüglich der Handlungsanreize besteht die Grundidee darin, daß Wirtschaftssubjekte, die nicht sicher sein können, daß die Früchte der eigenen Anstrengungen ihnen zufallen, geringe Anreize für Arbeitseinsatz und Investitionen haben. Zusätzlich werden der Planungshorizont und die Dauer der Investitionen kurzfristig angelegt sein. Bei vorhandenen exklusiven Eigentumsrechten bestehen dagegen spezifische Anreize für Investitionen und Arbeitseinsatz, wie z. B. die Erhaltung oder Erhöhung eines zukünftigen Einkommensstromes oder die Erhöhung des Bodenwertes. Für diesen Anreiz ist von Bedeutung, daß die Eigentümer auch über Verfügungsrechte und nicht nur über Nutzungsrechte verfügen, da der Bodenwert nur durch Verpachtung oder Verkauf realisiert werden kann. Was den zweiten Aspekt, Zugang zu Kredit, betrifft, wird in der Literatur betont, daß viele Investitionen in landwirtschaftliche Produktivität und Bodenschutz neben Arbeit Kapitaleinsatz verlangten. Es wird argumentiert, daß es wesentlich sei, über klare Eigentumsrechte sowie einen gesetzlichen Landtitel zu verfügen, um Land als Grundpfand einbringen zu können.

Mit Landrechtsregistrierung und Landtitelvergabe sollen die Probleme fehlender Handlungsanreize und mangelnden Zugangs zu Kredit vermindert und Anstrengungen für Bodenschutz verstärkt werden. Entsprechende Forderungen gehen zunehmend an die Entwicklungsbehörden und -institutionen (WORLD BANK 1992, 12). Empfehlungen gehen meist in Richtung verstärkter privater $\mathrm{Ei}$ gentumsrechte. Seit einigen Jahren wird diese Argumentation propagiert unter Verweis auf eine inzwischen berühmte und oft zitierte Evaluation eines Landtitulierungsprojektes der Weltbank in Thailand, wo die Effekte von individuellen Landtiteln auf die landwirtschaftliche Investitionstätigkeit untersucht wurden und wo ein signifikanter positiver Zusammenhang erkannt wurde (FEDER et al. 1988). Können diese Erkenntnisse und Empfehlungen jedoch als allgemein anwendbares Rezept dienen?

\section{Ist Informalität die Ursache von Besitzunsicherheit?}

Die Idee der Landtitulierung basiert auf der Annahme, daß das Fehlen eines offiziellen Landbesitznachweises die Ursache von Besitzunsicherheit sei. Ohne offiziellen Landtitel, der nötigenfalls durch den Staat abgesichert wird, regiere das Faustrecht. Dies ist allerdings eine verkürzte Sicht der Dinge. In vielen Gegenden der Dritten Welt, und insbesondere in Afrika, finden wir traditionelle informelle Landnutzungssysteme, die nach wie vor recht gut funktionieren. Dabei verfügen die einzelnen Bauern durchaus über verläßliche Sicherheit. Die Landnutzungssysteme sind häufig sehr flexibel und passen sich an sich verändernde demographische, wirtschaftliche oder ökologische Bedingungen an. Verschiedene Studien zei- gen, daß die Formalisierung hier u. U. wenig bringt bzw. sogar Schaden anrichten kann. Denn wenn in diesen traditionellen Gesellschaften das Verständnis für das formale Rechtssystem fehlt, kann es sein, daß Auswärtige sich in den Besitz der formellen Landrechte bringen ("Land Grabbing»), was zur Destabilisierung - auch im ökologischen Sinn - von ökologisch recht gut angepaßten Landnutzungssystemen führen kann (LAWRY 1990). Registrierung und Landtitel werden aber dann nötig, wenn durch Bevölkerungszunahme, Kommerzialisierung der Agrarwirtschaft und Auflösung der abgeschlossenen Dorfwirtschaften traditionelle Nutzungsregelungen aufgelöst werden. Hier entsteht ein Bedarf nach Formalisierung der Landrechte (WORLD BANK 1992, 20).

Dies ist übrigens auch eher die Situation, in der sich Osteuropa gegenwärtig befindet, mit den unklaren Eigentumsrechten nach dem Zusammenbruch des Kommunismus. Aber diese Situation darf nicht mit einem traditionellen, seit Jahrhunderten sich einspielenden afrikanischen Landnutzungssystem gleichgesetzt werden.

\section{Unterschiedliche Eigentumsrechtssituationen}

Vorschläge zur Landtitulierung gehen häufig von einem vereinfachenden Gegensatz «sichere, private Eigentumsrechte vs. fehlende, inexistente Eigentumsrechte» aus. Es ist aber zur Kenntnis zu nehmen, daß in Entwicklungsländern - unabhängig von der Frage «formell» oder «informell» - sehr unterschiedliche BesitzunsicherheitsProblemsituationen anzutreffen sind. Versuche, unterschiedliche Problemsituationen zu typisieren, sind natürlich mit dem Problem der Komplexität der realen Welt konfrontiert. Die hier verwendete Typologie knüpft an die neuere Literatur im Bereich der Umwelt- und Ressourcenökonomie über verschiedene Eigentumsrechtssysteme an (BROMLEY 1989). Traditionell unterschied man, wie bereits angetönt, einfach den Gegensatz zwischen privaten, exklusiven Eigentumsrechten und einem Zustand fehlender Eigentumsrechte. In der Realität gibt es aber eine Vielzahl von Bodenrechtssituationen: Staats-, Privat-, Gemeineigentum und eine Situation fehlender Eigentumsrechte («Nichteigentum»). Diese verschiedenen Eigentumsrechtssysteme sind in Abb. 2 dargestellt.

Die Besitzunsicherheitsproblematik ist unterschiedlich je nachdem, ob wir uns in einem Gebiet mit Privateigentum (z. B. in weiten Teilen Lateinamerikas), mit Gemeineigentum (z. B. traditionelles afrikanisches Landnutzungssystem) oder Staatseigentum (z. B. Staatsfarm in einem sozialistischen Land wie Äthiopien) befinden.

Die idealtypische Landtitulierungssituation, die auch den Vorschlägen zur Landtitulierung zugrunde liegt, wäre eine solche Situation, in der individuelle Bauern mit informellem Privatbesitz Land bebauen und dann einfach durch Registrierung in einen legalen Zustand übergeführt werden. Allerdings ist dies nur ein einfacher Fall. Wenn man die Landrechtsregistrierung und Landtitulie- 


\begin{tabular}{|ll|}
\hline Staatseigentum & $\begin{array}{l}\text { Die staatlichen Verwaltungsorgane } \\
\text { haben das Recht, Ressourcennut- } \\
\text { zungsregeln zu erlassen. Individuen } \\
\text { haben die Pflicht, die Nutzungsregeln } \\
\text { zu beachten. }\end{array}$ \\
Privateigentum & $\begin{array}{l}\text { Individuen haben das Recht auf Res- } \\
\text { sourcennutzung innerhalb der durch } \\
\text { das Recht gesetzten Schranken. }\end{array}$ \\
Nicht-Eigentümer haben die privaten \\
Rechte zu respektieren und sind von \\
der Nutzung ausgeschlossen. \\
Die Eigentümergruppe hat das Recht, \\
Nicht-Eigentümer von der Ressour- \\
cennutzung auszuschliessen. Nicht- \\
Eigentümer haben keinen Nutzungs- \\
anspruch. Die einzelnen Mitglieder \\
der Eigentümergruppe haben sowohl \\
Rechte als auch Pflichten in bezug \\
auf Nutzung und Unterhalt der Res- \\
source. \\
Es gibt keine definierte Gruppe von \\
Eigentümern. Jedermann hat Zutritt \\
zur Ressource, und es bestehen keine \\
Pflichten in bezug auf Nutzung und \\
Unterhalt.
\end{tabular}

Abb. 2 Vier Typen von Eigentumsrechtssystemen (Quelle: BROMLEY [1989, 872]; Übersetzung: DW).

rung in die Systematik der Eigentumsrechte von Abb. 2 einbezieht, gelangt man zu der bereits recht komplexen Matrix möglicher eigentumsrechtlicher Handlungsfelder in Abb. 3.

Abb. 3 muß von links her (Ausgangssituationen) nach oben (mögliche Endzustände) gelesen werden. Man kann sich also beispielsweise von einer informellen privaten Ausgangssituation zu einem formellen privaten Endzustand bewegen (Feld 1) oder von einem informellen Gemeineigentumssystem zu einer formellen privaten Eigentumsrechtssituation (Feld 4), was die Privatisierung eines Gemeineigentumssystems bedeutet. In Abb. 3 fehlt die Kategorie «Nichteigentum», denn bei den Ausgangssituationen geht es nicht um Rechtslagen, sondern Interessen, wozu private, gemeinschaftliche oder staatliche Interessenten benötigt werden. Und bei den Endzuständen soll Nichteigentum gerade vermieden werden. Da hier nicht auf alle eigentumsrechtlichen Handlungsfelder eingegangen werden kann, konzentriert sich dieser Abschnitt auf einen ganz bestimmten Problemkreis, der für weite Teile der Dritten Welt von beträchtlicher Bedeutung ist und der die konventionelle Eigentumsrechtsund Landtitelargumentation herausfordert, nämlich auf Probleme in Gemeineigentumssystemen, die in vielen traditionellen Gesellschaften der Dritten Welt anzutreffen sind (Abb. 3: Zeile «Gemeinschaftlich»). In den vergangenen Jahren hat sich eine recht breite Literatur zu anderen als Privateigentumsrechtssystemen, insbesondere Gemeineigentumssystemen, entwickelt, (CIRIACY-WANTRUP / BISHOP 1975, BROMLEY / CERNEA 1989). In der umweltökonomischen Literatur wurde Gemeineigentum lange mit einer Situation fehlender Eigentumsrechte gleichgesetzt. HARDIN (1968) prägte den Begriff «Tragedy of the Commons"; er argumentierte, daß knappe Ressourcen bei fehlenden privaten Eigentumsrechten übernutzt werden, da die einzelnen Wirtschaftssubjekte sich in einem Gefangendilemma befinden und untereinander keine Nutzungsbeschränkungen beschließen können. Diese Begrifflichkeit hat zu einer Diskreditierung von solchen Gemeineigentumssystemen geführt, die aus einer klar abgegrenzten Gruppe autorisierter Nutzungsberechtigter bestehen, die das Recht haben, Dritte von der Nutzung auszuschließen.

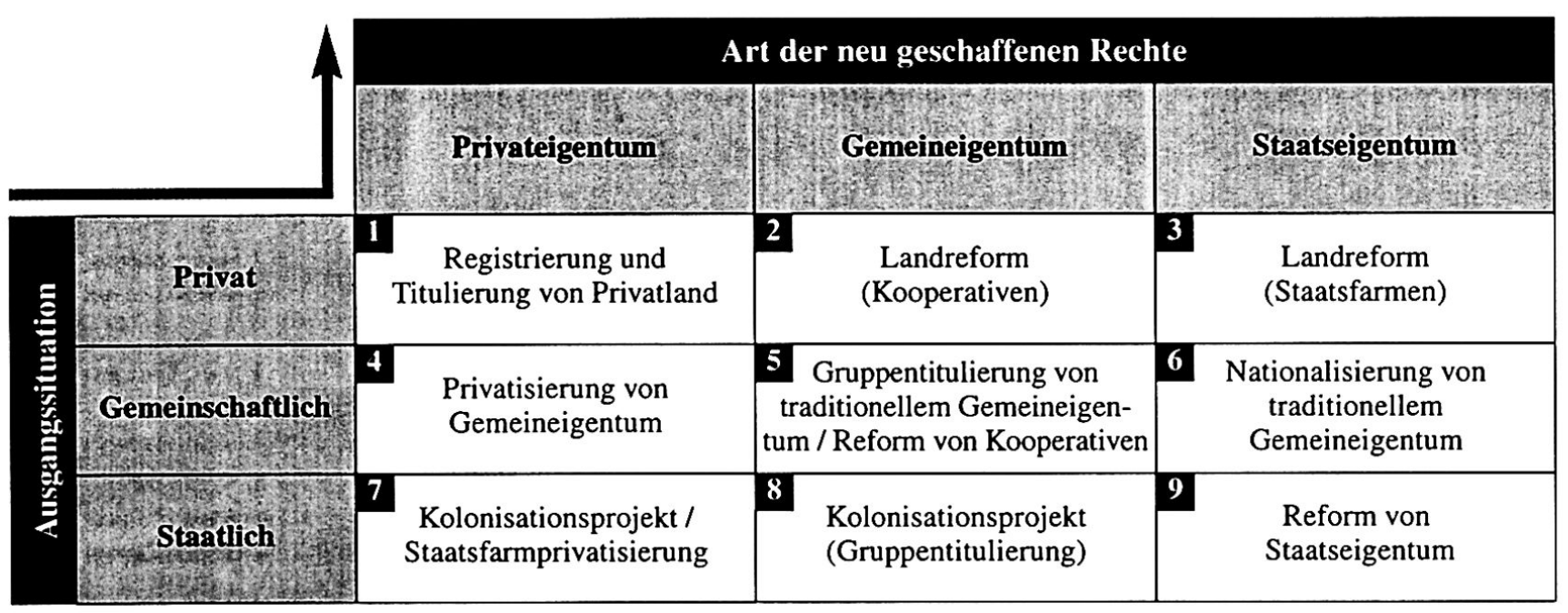

Abb.3 Typen von Landtitulierungsprojekten (Quelle: WACHTER [1992, 29]). 
Gemeineigentumssysteme, die häufig informelle Rechtssysteme sind, sind weit verbreitet in Schwarzafrika sowie in traditionellen Gesellschaften in Lateinamerika und Asien. Auch in Industrieländern sind noch Gemeineigentumssysteme anzutreffen, wo die erzwungenen Privatisierungsprozesse zu Beginn der Neuzeit nicht voll zum Tragen kamen (z. B. alpine Weidewirtschaften). Diese traditionellen Gemeineigentumssysteme dürfen nicht mit kollektivistischen Kooperativen verwechselt werden. Solche Systeme wurden bis vor kurzem von der westlichen Wissenschaft, von Kolonialverwaltern usw. unkorrekterweise als statische Gegensätze zu westlichen Eigentumssystemen dargestellt (MIGOT-ADHOLLA et al. 1991). Es wird oft behauptet, die Landrechte seien der Gemeinschaft als solcher zugewiesen und die individuellen Bauern hätten deshalb keine Anreize für Investitionen und Arbeitseinsatz. Es wird jedoch oft übersehen, daß die einzelnen Bauern oder Familien über recht sichere Nutzungsrechte verfügen. Ausgeklügelte Nutzungsregelungen mit Rechten und Pflichten sorgen dafür, daß die gemeinschaftlichen Ressourcen nicht übernutzt werden. Auch sind im allgemeinen Landtransaktionen durchaus möglich, wenn auch der Handel mit Landrechten nicht selten auf den Stamm oder die erweiterte Familie beschränkt ist. Auch haben sich viele dieser Systeme als recht flexibel für die Anpassung an veränderte sozioökonomische Bedingungen erwiesen (NORONHA 1985).

Sind solche Gemeineigentumssysteme wegen der angeblichen Besitzunsicherheit in Privateigentumssysteme zu transformieren? Um diese Frage beantworten zu können, brauchen wir ein noch besseres Verständnis der Merkmale und der Funktionsweise von traditionellen Gemeineigentumssystemen, was Zweifel bezüglich der Nützlichkeit von Privatisierungsanstrengungen aufkommen lassen wird. Man sollte sie nicht vorschnell aus ideologischen oder ethnozentrischen Gründen disqualifizieren. Vielmehr ist zur Kenntnis zu nehmen, daß diese Institutionen in Anpassung an lokale Bedürfnisse und Bedingungen entstanden sind. Die weitverbreitete Unfähigkeit und fehlende Bereitschaft, die möglichen Vorteile von Gemeineigentumssystemen zu verstehen, hat oft $\mathrm{zu}$ unzweckmäßigen Politikeingriffen geführt.

Es wurde oben erwähnt, daß die einzelnen Mitglieder von traditionellen Gemeineigentumssystemen im allgemeinen sichere individuelle Nutzungsrechte haben, auch wenn diese Rechte häufig informeller Natur sind, und daß ausgeklügelte Nutzungsregelungen der Ressourcendegradation entgegenwirken. Ferner werden in der Literatur mehrere Argumente für Gemeineigentumslösungen erwähnt (WACHTER 1992, 44 f.):

- Arbeitsmethoden oder Technologien mit gemeinsamen Kosten oder Externalitäten, d. h., daß mehrere Personen Nutzen daraus ziehen können, Nutznießer und Kostenträger also nicht genau abgegrenzt werden können (z. B. komplexe Bewässerungssysteme).

- Ein im Verhältnis zu den Kosten privater Eigentumsrechte geringer Nutzen, d. h., daß die Kosten der Abgrenzung und Durchsetzung privater Eigentums- rechte sich möglicherweise nicht lohnen, wo die Produktivität gering ist (z. B. eine extensive Weide).

- Ökologische Faktoren: In ariden oder semiariden Weidegebieten mit zeitlich und räumlich variierenden Niederschlägen beispielsweise erlaubt Gemeineigentum die relativ leichte Verschiebung von Herden hin zu Gebieten mit günstigen Weidebedingungen.

Man darf allerdings auch nicht den Fehler der Romantisierung dieser Eigentumssysteme machen. Sie sind vielfältigen Belastungen ausgesetzt, und verschiedene der genannten Faktoren verlieren an Bedeutung und können das Gewicht zugunsten privater Eigentumsrechte verschieben. Im Zuge der allgemeinen Veränderungen der Gesellschaften in den Entwicklungsländern nimmt in vielen Situationen die wirtschaftliche Bedeutung der lokalen Ressourcen ab. Andere, meist nichtlandwirtschaftliche Einkommensquellen gewinnen auch in ländlichen Gebieten an Bedeutung. Auch Geldüberweisungen von Verwandten in Städten können zu diesen Veränderungen beitragen. Dadurch vermindern sich die Anreize für die Individuen, sich aktiv im Gemeineigentumssystem zu engagieren. Zudem vermindert die allgemeine Öffnung der Dorfwirtschaften den Respekt für traditionelle Autoritäten und erschwert dadurch die interne Organisation von Gemeineigentumssystemen, das Aufrechterhalten von Loyalitäten und die Erlangung von Übereinkünften bezüglich der Ressourcennutzung (LAWRY 1990).

Was bedeutet all dies für die Landtiteldiskussion? Die zentrale Schlußfolgerung ist, daß die Realität von traditionellen Gemeineigentumssystemen komplex ist und nicht einfach nach einer Transformation in private Eigentumssysteme ruft. Es besteht die Gefahr, daß Umweltund Landbehörden aus ideologischen oder ethnozentrischen Gründen solche Systeme "reformieren» wollen. "Prophylaktische» Landrechtsreformen, oft mit der impliziten oder expliziten Absicht, den Entwicklungsprozeß zu beschleunigen, sind meist fehlgeschlagen. Landtitelvergabe und Bodenbesitzregistrierung werden dagegen dann notwendig, wenn Unsicherheiten über die Anwendbarkeit und Zweckmäßigkeit traditioneller Nutzungsregelungen und -kontrollen erwachsen, wo die Knappheit des Bodens, der Bodenwert, die Ansprüche an den Boden und die Konflikte zunehmen und wo die Bevölkerung selber die Notwendigkeit der Formalisierung des Landrechtssystems verspürt.

Allerdings sind nicht notwendigerweise individuelle Landtitel erforderlich. Eine potentielle Alternative sind Gruppentitel, insbesondere wenn das Hauptproblem nicht die interne Organisation des Gemeineigentumssystems ist, sondern die Gefährdung durch Außenstehende. Gruppentitel sind auch zu prüfen, wo technische oder ökologische Faktoren (z. B. in der Weidewirtschaft räumlich variierende Weideproduktivität wegen räumlich variabler Regenfälle) gemeinschaftliche Lösungen auch unter veränderten sozioökonomischen Bedingen aufdrängen. 


\section{Notwendige, aber nicht hinreichende Besitzsicherheit}

Sehen wir jetzt wieder ab von den unterschiedlichen Eigentumsrechtssituationen und kehren zurück zur idealtypischen Situation im Feld 1 (Abb. 3). Jetzt stellt sich die Frage, ob Besitzsicherheit den Landbewirtschaftern wirklich hinreichende Anreize bezüglich Bodenschutz gibt. Dies hängt stark von der Frage ab, ob andere schädliche Handlungsanreize vorliegen. Die Erfahrung aus den Industrieländern, wo die Landrechte im allgemeinen sicher sind, die Bauern aber dennoch beträchtliche Bodenschädigungen verursachen, zeigt, daß klare und durchsetzbare Landrechte allein nicht Bodenschutz garantieren. In einem irreführenden Politikumfeld können die Anreize, die eindeutige Landrechte vermitteln, zu unerwünschten Resultaten führen. Unser Wissensstand über umweltschädigende Handlungsanreize in Entwicklungsländern ist heute beträchtlich, insbesondere im Bereich der Agrarpolitik. Unzählige Regierungspolitiken in vielen Ländern sind nicht nur unfähig, die realen Kosten von Land- und Naturnutzung den Wirtschaftssubjekten anzulasten, sondern fördern sogar aktiv eine stärkere Umweltzerstörung, als sie ohne Staatseingriffe zustande käme. Politiken werden verfolgt, die künstlich die Rentabilität von umweltbelastenden Aktivitäten erhöhen. Diese Verzerrungen umfassen eine Vielzahl von Subventionen und Markteingriffen (PEARCE/WARFORD 1993, $173 \mathrm{ff}$.). Für die vorliegende Problematik von besonderer Relevanz sind manipulierte Preise von Agrarerzeugnissen und landwirtschaftlichen Inputs. Was die Agrarpreise betrifft, sind in Entwicklungsländern durch überbewertete Wechselkurse oder Preisfestsetzungen künstlich niedrig gehaltene Erzeugnispreise von erstrangiger Bedeutung. Dadurch werden die internen Tauschverhältnisse zuungunsten der Landwirtschaft verzerrt. Eine künstlich niedrig gehaltene landwirtschaftliche Rentabilität reduziert die Anreize für Investitionen in landwirtschaftliche Produktivität und Bodenschutz.

Hier tragen natürlich auch diejenigen Industrieländer Verantwortung, die ihre Landwirtschaft stark subventionieren und dadurch Agrarüberschüsse verursachen, was die Weltmarktpreise für viele Landwirtschaftsgüter reduziert.

Was verzerrte Inputpreise betrifft, subventionieren viele Regierungen z. B. Wasser für Bewässerung (was ineffizienten Wassereinsatz und Versalzung begünstigt), Pestizide (was Flora und Fauna sowie die menschliche Gesundheit schädigt) oder Dünger (was zu Überdüngung führt).

\section{Landtitulierung als Mittel gegen Bodendegradation?}

Zur Erhöhung der Besitzsicherheit werden, wie eingangs erwähnt, immer wieder Landtitulierungsprojekte vorgeschlagen. Sind diese tatsächlich die Lösung? Es soll an dieser Stelle nicht mehr auf die Problematik unterschiedlicher Eigentumsrechtssysteme eingegangen werden. Vielmehr nehmen wir jetzt an, wir hätten ein Gebiet vor uns mit Kleinbauern mit informellem Privatbesitz, also eine idealtypische Landtitulierungssituation (Feld 1 in Abb. 3).

Bauern beurteilen Landtitel hinsichtlich des Verhältnisses von Nutzen und Kosten. Vieles hängt davon ab, wie sich diese Kosten und Nutzen konkret präsentieren (WACHTER 1992, 55 ff.). Wenn die Kosten von Landtiteln aus Sicht der Bauern den Nutzen übersteigen, ist es für sie lohnender, mit informellen Landrechten weiter zu operieren und eventuell Bodendegradation hinzunehmen. Es geht hier um die Geld- und Zeitkosten im Zusammenhang mit der Erlangung, der Aufrechterhaltung und auch der Veräußerung von Landtiteln. Eine ungenügende Landverwaltung und eine ineffiziente Bürokratie können diese Handlungen äußerst komplex, mühsam und teuer bezüglich Geld und Zeitaufwand machen.

Das Problem kann auch aus dem Blickwinkel des Nutzens eines Landtitels betrachtet werden. Theoretisch besteht der Zweck eines Landtitels ja darin, die Sicherheit des Bodenbesitzes zu erhöhen und Zugang zu Kredit zu verschaffen, um damit Anreize und Befähigung für Bodenschutz zu erhöhen. Von ganz entscheidender Bedeutung ist jetzt, ob ein Landtitel effektiv Zugang zu Kredit verschafft. Dies ist in der Realität häufig nicht der Fall, weil die Banken Kleinbauern oft keinen Kredit geben, auch wenn sie über einen Landtitel verfügen (WACHTER 1992 a). Ein generelleres Problem ist das institutionelle Umfeld im Sinne der allgemeinen politischen und bürokratischen Rahmenbedingungen von wirtschaftlichen Handlungen. Viele Staaten in der Dritten Welt sind durch institutionelle Schwäche, Instabilität und Unsicherheit gekennzeichnet. Regierungen wechseln häufig von einem Tag auf den anderen; Präsidenten regieren auf dem Dekretweg ohne Rechenschaftspflicht. Willkür und Instabilität sind für die vorliegende Fragestellung von Bedeutung, weil in einem unsicheren Umfeld (z. B. Angst vor Enteignung) die Funktion eines Landtitels vermindert wird.

\section{Schlußbemerkungen}

Landtitelvergabe zur Förderung von Bodenschutzaktivitäten ist eine Idee, die aus der Eigentumsrechtsökonomie abgeleitet wurde. Sie wird vor allem von gewissen politischen Gruppen, die auf eine reduzierte Rolle des Staates in der Wirtschafts- und Umweltpolitik abzielen, als eine einfache und vielversprechende Maßnahme propagiert. Es sollte jedoch aufgrund dieser Ausführungen klargeworden sein, daß die Besitzunsicherheitsproblematik äußerst komplex ist. Erfolg und Mißerfolg von Landtitelprogrammen hängen stark von den Bedingungen (Eigentumsrechtssituationen) und der Art und Weise der Umsetzung ab. Landtitulierung ist nicht überall nötig. Wo diese Maßnahme als notwendig erachtet wird, hängt der Erfolg von Faktoren wie der Reform von Agrarpolitiken, Reform der öffentlichen Verwaltung usw. ab. Landtitelprogramme können nur Teil einer umfassenden Entwicklungsstrategie sein. 


\section{Literatur}

ANDERSON, J. / THAMPAPILLAI, J. (1990): Soil Conservation in Developing Countries - Project and Policy Intervention. Washington, D. C. (= World Bank, Policy and Research Series, Nr. 8).

BROMLEY, D. W. (1989): Property Relations and Economic Development: the Other Land Reform. In: World Development, 17(6), S. 867-877.

BROMLEY, D. W. / CERNEA, M. M. (1989): The Management of Common Property Natural Resources. Washington, D. C. (= World Bank Discussion Paper Nr. 57).

BUHBE, M. (1985): Ökonomische Analyse von Eigentumsrechten. Frankfurt am Main.

CHISHOLM, A. / DUMSDAY, R. (Hrsg.) (1987): Land Degradation - Problems and Policies. Cambridge.

CIRIACY-WANTRUP, S. / BISHOP, R. (1975): "Common Property" as a Concept in Natural Resource Policy. In: Natural Resources Journal, 15(4), S. 713-727.

FEDER, G. / FEENY, D. (1991): Land Tenure and Property Rights: Theory and Implications for Development Policy. In: World Bank Economic Review, 5(1), S. 135-153.

FEDER, G. / ONCHAN, T. / CHALAMWONG, Y. / HONGLADAROM, C. (1988): Land Policies and Farm Productivity in Thailand. Baltimore / London.

HARDIN, G. (1968): The Tragedy of the Commons. In: Science, 162 , S. $1243-1248$.

JOHNSON, O. E. G. (1972): Economic Analysis, the Legal Framework and Land Tenure Systems. In: Journal of Law and Economics, 15(April), S. 259-276.
LAWRY, S. W. (1990): Tenure Policy Toward Common Property Natural Resources in Sub-Saharan Africa. In: Natural Resources Journal, 30 (Spring), S. 403-422.

MIGOT-ADHOLLA, S. / HAZELL, P. / BLAREL, B. / PLACE, F. (1991): Indigenous Land Rights Systems in Sub-Saharan Africa: a Constraint on Productivity? In: World Bank Economic Review, 5(1), S. 155-175.

NORONHA, R. (1985): A Review of the Literature on Land Tenure Systems in Sub-Saharan Africa. Washington, D. C. (= World Bank, Agriculture and Rural Development Department Discussion Paper Nr. 43).

PEARCE, D. W., WARFORD, J. J. (1993): World Without End: Economics, Environment and Sustainable Development. Washington, D. C.

SOUTHGATE, D. (1988): The Economics of Land Degradation in the Third World. Washington, D. C. (= World Bank, Environment Department Working Paper Nr. 2).

WACHTER, D. (1992): Farmland Degradation in Developing Countries: The Role of Property Rights and an Assessment of Land Titling as a Policy Intervention. Madison-Wisconsin (= Land Tenure Center Paper Nr. 145).

WACHTER, D. (1992 a): Die Bedeutung des Landtitelbesitzes für eine nachhaltige landwirtschaftliche Bodennutzung eine empirische Fallstudie in Honduras. In: Geographische Zeitschrift, 80(3), S. 174-183.

WORLD BANK (1992): World Development Report 1992 (Development and the Environment). Washington, D. C. 